\title{
Vorwort zur 1. Auflage
}

\begin{abstract}
Je gründlicher ein Massör ausgebildet ist, um so weniger neigt er zu Kurpfuscherei. Je mehr er sieht und lernt, desto mehr erkennt er die Gefahren für die Patienten, wie für sich, wenn er ohne ärztliche Anordnung arbeitet.
\end{abstract}

KIRCHBERG

Dieses Buch ist aus dem Bedürfnis der Praxis entstanden. Bei den zahlreichen Kursen zur Ausbildung von Massören an einer staatlich anerkannten Massageschule und auch bei den Fortbildungskursen für Massöre wurde immer wieder der Wunsch vorgebracht, ein Lehrbuch über das ausgedehnte Material, welches in den Kursen geboten wurde, zu besitzen.

Und so ist dieses Buch für Massöre geschrieben. Einmal für die Schüler an den staatlich anerkannten Massageschulen als notwendiges Hilfsmittel des Unterrichts, zum anderen auch als Nachschlagewerk für die Massöre in der Praxis.

Es soll dabei kein Lehrbuch sein zum Erlernen der Massage; diese Aufgabe muß immer den praktischen Kursen vorbehalten bleiben.

Andererseits mußte besonders ausführlich jener Teil über den Aufbau und die Funktionen des menschlichen Körpers nebst der Krankheitslehre behandelt werden. Im Gegensatz zu den Lehrbüchern für Krankenpfleger und ärztliches Hilfspersonal wurde hier immer die spezielle Aufgabe im Auge behalten, alles das bevorzugt zu bringen, was der Massör bei der Ausführung seiner Aufgaben besonders benötigt. So wurde der Muskel- und Nervenlehre naturgemäß ein viel größerer Platz eingeräumt als es sonst in diesen Lehrbüchern üblich ist. Und hierzu war auch ein ausreichendes Bildmaterial nötig für das Verständnis und das bessere Einprägen des Vorgebrachten.

Deshalb bin ich Herrn Professor Dr. Dr. A. WALdeyer, Berlin, besonders dankbar, daß er mir eine große Anzahl der hier veröffentlichten anatomischen Bilder aus seinem Werke $^{1}$ ) überließ.

Im praktischen Teil wurde auf eine Darstellung der typischen Massagegriffe im Bild verzichtet. Dagegen gelang es, durch Einführung von Trickzeichnungen die Darstellung einzelner Bewegungsübungen anschaulich zu gestalten. Im übrigen soll hier nur das in den Kursen Erlernte für das Gedächtnis festgehalten werden und nicht etwa soll der praktische Unterricht ersetzt werden.

1) A. WALDEYER, Anatomie des Menschen Band I, 3. Auflage 1956 und Band II, 1. Auflage 1950. 
Bei der Abfassung dieses praktischen Teiles bin ich meiner Frau, langjährige Mitarbeiterin und Lehrerin in diesen Massagekursen, Ursula Thulcke geb. Scholz, welche an der Ausarbeitung dieses Teiles hervorragenden Anteil hatte, zu großem Dank verpflichtet.

In diesem praktischen Teil ist der Aufbau der Massage und der Bewegungsübungen ausführlich beschrieben. Dagegen konnte die Anwendung der Massage und Gymnastik bei den einzelnen Krankheiten nur in kurzer Form gebracht werden, da sie über den Umfang der gestellten Aufgabe hinausgehen würde.

Andererseits jedoch wurde die elektrophysikalische Behandlung in jener Form, wie sie bei uns in einem Elektrokurs gelehrt wird, mit aufgenommen. Ist doch eine ausreichende Kenntnis auf diesem Gebiet für den Massör bei seiner täglichen Arbeit unbedingt notwendig. Für die Durchsicht des physikalischen Teiles bin ich Herrn Oberingenieur OBERHAUSER zu Dank verpflichtet.

Und so hoffe ich, daß dieses Buch, entstanden aus dem Wunsch der Massöre, ihnen bei der Ausbildung und weiteren Fortbildung eine gute Hilfe sein wird.

Berlin, im Herbst 1955

Erich Thulcke 